\title{
Exploiting Gynoecious Line for Earliness and Yield Traits in Bitter Gourd (Momoredica charantia L.)
}

\author{
Ahmad Alhariri $^{*}$, T.K. Behera ${ }^{1}$, A.D. Munshi ${ }^{1}$, C. Bharadwaj ${ }^{2}$ and G.S. Jat ${ }^{1}$ \\ ${ }^{1}$ Division of Vegetable Science, ${ }^{2}$ Division of Genetics, IARI, New Delhi 110012, India \\ *Corresponding author
}

\begin{abstract}
Keywords
Bitter gourd,

Heterosis,

Gynoecious,

Earliness, Yield

Article Info

Accepted:

10 October 2018

Available Online:

10 November 2018 gynoecious line as one parents were found to be superior in performance over mid and standard parents for several earliness and yield characters. The best $\mathrm{F}_{1}$ hybrid for earliness and desired sex ratio was PDMGy-201 $\times \mathrm{S}-2$ which recorded $-32.58 \%$ heterosis for days to opening of first female flower over mid parent and $-35.96 \%$ over standard parent. This hybrid also showed $-23.65 \%$ heterosis for days to first picking over mid parent and $28.24 \%$ over standard parent. The hybrid PDMGy-201 $\times$ S-2 showed $-95.93 \%$ and $69.83 \%$ heterosis for sex ratio (male: female) over mid and standard parent respectively. The monoecious hybrid, S-54 $\times$ S-57 showed higher heterosis for fruit length (33.43\%) over mid parent, for fruit diameter $(15.12 \%)$ over standard parent and for flesh thickness $(54.88 \%)$ and $(29.07 \%)$ over mid and standard parent respectively. All the $28 \mathrm{~F}_{1}$ crosses exhibited significant desirable heterosis for average fruit weight and yield per plant over mid and standard parent. The best performing $\mathrm{F}_{1}$ hybrid PDMGy-201 $\times$ PV recorded $67.5 \%$ and $67.22 \%$ higher yield over mid and standard parent and $18.92 \%$ increase in number of fruits per plant over standard parent which may be exploited for commercial cultivation.
\end{abstract}

\section{A B S T R A C T}

Eight parental lines including one gynoecious line PDMGy-201 (Gy-23) and their $28 \mathrm{~F}_{1}$ hybrids obtained from half diallel analysis were used to estimate the extent of heterosis for earliness, yield and its contributing traits in bitter gourd. The $\mathrm{F}_{1}$ hybrids developed using

\section{Introduction}

Bitter gourd (Momordica charantia L.) is a tropical and subtropical cucurbitaceous vegetable crop grown extensively in India, China, Japan, South East Asia, Tropical Africa and South America. Among all cucurbits, it is considered as prized vegetable because of its tender fruits have high nutritive values especially ascorbic acid and iron (Behera, 2004). Fruits and seeds of bitter gourdare not only used for its hypoglycemic properties, but also against cancer, malaria, ulcer, dyslipidemia, and hypertension (Alam et al., 2015) with its anti-inflammatory, antiviral, and antibacterial properties (Budrat et al., 2008; Joseph et al., 2013). Due to efforts of vegetable breeders, several improved varieties and hybrids have been developed (Laxuman et al., 2012) in India. Being a cross-pollinated crop and it has high levels of heterozygosity and hence, heterosis is well exploited in bitter 
gourd for early harvest, higher yield and other agronomic traits. The predominance of dominant gene action coupled with low heritability observed for most of yield traits indicating the importance of heterosis breeding for improvement of yield and yield attributing traits in bitter gourd (Radha Rani et al., 2014). Monoecious is the predominant sex form in bitter gourd, however, gynoecious sex form has been reported from India, Japan and China (Behera et al., 2006). Gynoecism is under the control of a single recessive gene (gy-1) (Ram et al., 2006; Behera et al., 2009). There is a paramount need to develop suitable hybrids, which may be utilized on commercial scale especially in the north Indian plains. However, the utilisation of gynoecy is economical and easier for exploiting hybrid vigour in many cucurbits including bitter gourd (Behera, 2004) that have high male: female sex ratio. Using gynoecious line as one parent in hybrid breeding, it showed positive impact in terms of yield and earliness (Dey et al., 2010; Gangadhara Rao et al., 2018). Keeping in view the above facts, the present investigation was carried out to examine the extent of heterosis in $28 \mathrm{~F}_{1}$ hybrids by using eight diverse parents.

\section{Materials and Methods}

The study was conducted at the Research Farm of Division of Vegetable Science, IARI, New Delhi, during spring-summer (February to May), 2016-17. Eight genetically diverse inbred lines/cultivars of bitter gourd including one gynoecious line, PDMGy-201and seven monoecious parents, S-54, S-2, Pusa Do Mausami (PDM), Pusa Vishesh (PV), S-32, Pusa Aushadhi (PA) (predominantly gynoecious) and S-57 were crossed with all possible combinations $\left(28 \mathrm{~F}_{1} \mathrm{~s}\right)$ excluding reciprocals by following half diallel fashion (Hayman, 1954) during spring summer, 201516. The $28 \mathrm{~F}_{1}$ hybrids along with parents were evaluated in a randomized block design
(RBD) with three replications to estimate the heterosis. The bitter gourd crop was grown in channels at $2.0 \mathrm{~m}$ apart with spacing of $0.50 \mathrm{~m}$ between the plants. All the recommended cultural practices for irrigated conditions were followed to raise a healthy and successful crop. The gynoecious lines were maintained by spraying silver thiosulphate @ 3mM at different growth stages. Observations were recorded on five randomly tagged plants in each entry for node number to first female flower, days to opening of first female flower, days to first picking, sex ratio (male: female), fruit length $(\mathrm{cm})$, fruit diameter $(\mathrm{cm})$, flesh thickness $(\mathrm{mm})$, number of fruits per plant, average fruit weight $(\mathrm{g})$ and yield per plant $(\mathrm{kg})$. The heterosis was calculated as the percentage of $F_{1}$ performance in the favourable direction of mid parent and standard check (Pusa Vishesh) for each character.

\section{Results and Discussion}

The analysis of variance revealed highly significant differences among the experimental material indicating high genetic variation for most of the characters. The per cent heterosis over mid parent (MP) and standard check (Pusa Vishesh) and the range of mean values for different traits of parents, $\mathrm{F}_{1}$ hybrids and heterosis (over mid and standard parent) are presented in Table 1, 2 and 3 , respectively. The results indicated that there was wide variation in magnitude and direction of heterosis for all the traits.

Among the parents, gynoecious line PDMGy201 was the earliest and took minimum days to first female flower opening (37), days to fruit harvest (57) with lowest node number to first female flower (4.67) and sex ratio (100\% female). The monoecious parent S-2 recorded maximum fruit length $(17.97 \mathrm{~cm})$ and second best parent for average fruit weight $(60.7 \mathrm{~g})$ and yield per plant $(2.1 \mathrm{~kg})$. Highest number 
of fruits per plant (40.33) and fruit diameter $(4.83 \mathrm{~cm})$ was recorded in $\mathrm{P}_{2}(\mathrm{~S}-54)$, whereas predominantly gynoecious line (Pusa Aushadhi) reported the maximum yield per plant $(2.31 \mathrm{~kg})$ and number of fruits per plant similar to S-54 (40.33).

The mean range of different traits of $F_{1}$ hybrids were desirably higher than those of the parents for fruit diameter, flesh thickness, number of fruits per plant, average fruit weight and yield per plant, while the mean values of hybrids were desirably lower than those of the parents for days to first female flower opening, days to first fruit harvest, sex ratio $(\mathrm{M} / \mathrm{F})$ and fruit length (Table 3$)$. The present study revealed significant heterosis in positive and negative directions for all the characters.

The range of heterosis for $\mathrm{F}_{1}$ hybrids varied from (-15.89 to $106.06 \%)$ and $(-51.34$ to $59.53 \%$ ) for the number of node bearing first female flower, (-32.58 to $5.45 \%)$ and $(-35.96$ to $5.76 \%$ ) for days to appearance of first female flower, $(-23.65$ to $6.70 \%)$ and $(-28.24$ to $3.71 \%)$ for days to first harvest, $(-95.93$ to $34.78 \%)$ and (-99.07 to $51.46 \%)$ for sex ratio, $(-23.92$ to $33.43 \%)$ and $(-17.04$ to $47.62 \%)$ for length of fruits, (-6.84 to $40.70 \%)$ and (30.02 to $15.12 \%)$ for diameter of fruit, $(-3.66$ to $44.88 \%)$ and (-18.26 to $29.07 \%)$ for fruit flesh thickness, (-10.92 to 22.03\%) and (18.03 to $18.92 \%$ ) for number of fruits per plant, (-6.68 to $75.83 \%)$ and (15.19 to $60.67 \%$ ) for average weight of fruit, (9.81 to $67.50 \%$ ) and (16.11 to $68.33 \%$ ) for average yield per plant over mid parent and standard parent respectively.

Earliness is a critical consideration of any breeding programme as it helps the grower to maximize the financial gain by fetching higher market price. Earliness (indicated by negative estimates of heterosis) in bitter gourd is attributed to node number to first female flower, time required for first female flower appearance and time for first fruit harvest. Out of $28 \mathrm{~F}_{1}$ crosses 24 and 19 crosses exhibited significant negative heterosis for days to first female flower over mid and standard parent respectively.

For days to first fruit harvest, out of $28 \mathrm{~F}_{1}$ crosses 20 and 23 crosses exhibited significant negative heterosis over mid and standard parent respectively.

In the present findings it was found that gynoecious $\times$ monoecious hybrids were the most promising for earliness. In order of merit, three crosses namely PDMGy-201 x S2, PDMGy-201 × PDM and PDMGy-201 x S32 were found to be best performing hybrids for days to opening of first female flower and these showed significant heterosis of -32.58 , 25.6, and $-14.18 \%$ over mid parent and -35.96 , -33.09 and $-25.9 \%$ over standard parent respectively. The maximum negative heterosis for days to first harvest was also observed in gynoecious $\times$ monoecious hybrids and PDMGy-201 × S-2 showed $-23.65 \%$ heterosis over mid parent and $-28.24 \%$ heterosis over standard parent, PDMGy-201 × PV $-17.53 \%$ heterosis over mid parent and $-25.93 \%$ heterosis over standard parent.

This might have resulted due to the transfer of earliness character from the gynoecious parent to the hybrid. whereas, only $3 \mathrm{~F}_{1}$ hybrids over mid parent and 10 over standard parent showed significant negative hetrosis for nodes to first female flower. Lowest number of nodes to first female flower recorded in the cross S-2 $\times$ S-32 (-15.89\%) followed by PDM $\times$ S-32 $(-8.16 \%)$ and S-54 $\times$ PA $(-5.26 \%)$ over mid parent and S-54 $\times$ PA) $(-51.34 \%)$ followed by PDMGy-201 × S-54 (-27.01\%) and S-54 $\times$ PV $(-21.57 \%)$ over standard parent. In general, the higher the proportion of female flower greater is the productivity. 
Int.J.Curr.Microbiol.App.Sci (2018) 7(11): 922-928

Table.1 Heterosis (\%) over mid parent and standard check for flowering traits in bitter gourd

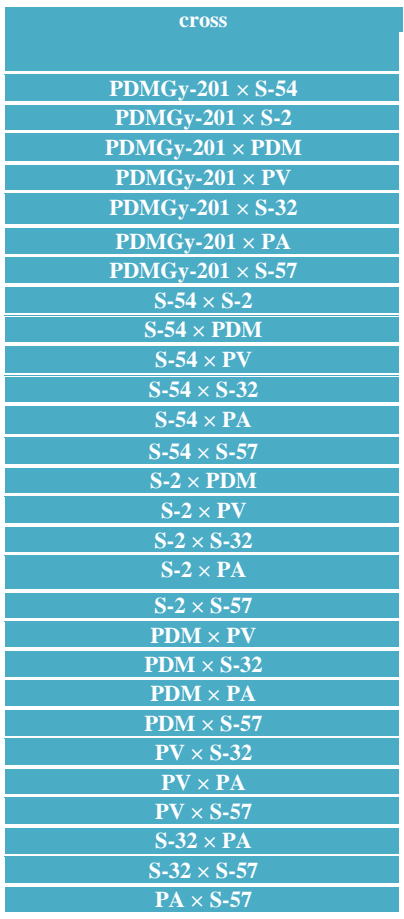

\begin{tabular}{|c|c|c|c|c|c|c|c|}
\hline \multicolumn{2}{|c|}{ NFF } & \multicolumn{2}{|c|}{ DFF } & \multicolumn{2}{|c|}{ DFH } & \multicolumn{2}{|c|}{ Sex ratio $\mathrm{m} / \mathrm{f}$} \\
\hline MPH & SPH & MPH & SPH & MPH & SPH & MPH & SPH \\
\hline $63.64 * *$ & $-27.01 * *$ & $-12.71^{* *}$ & $-25.90^{* *}$ & $-4.55^{* *}$ & $-22.22 * *$ & $-54.84 * *$ & $-81.38 * *$ \\
\hline $16.67^{* *}$ & $-5.35^{* *}$ & $-32.58 * *$ & $-35.96 * *$ & $-23.65 * *$ & $-28.24 * *$ & $-95.93 * *$ & $-96.81 * *$ \\
\hline $49.02 * *$ & 2.76 & $-11.02 * *$ & $-18.69^{* *}$ & $-9.66^{* *}$ & $-19.90 * *$ & $-57.52 * *$ & $-73.27 * *$ \\
\hline $21.57^{* *}$ & $-16.22 * *$ & $-25.60^{* *}$ & $-33.09 * *$ & $-17.53 * *$ & $-25.93 * *$ & $-44.75^{* *}$ & $-72.34 * *$ \\
\hline $4.00 * *$ & $5.43 * *$ & $-14.18^{* *}$ & $-19.43 * *$ & $-7.89 * *$ & $-18.99 * *$ & $-35.91 * *$ & $-61.84 * *$ \\
\hline $106.06^{* *}$ & $-8.11^{* *}$ & -2.56 & $-17.98^{* *}$ & -1.89 & $-15.74 * *$ & $34.78 * *$ & $-99.07 * *$ \\
\hline $50.00^{* *}$ & $21.65^{\text {** }}$ & $-11.45^{* *}$ & $-16.53^{* *}$ & -0.26 & $-11.57 * *$ & $-71.24 * *$ & $-77.53 * *$ \\
\hline $4.62 * *$ & $-8.11^{* *}$ & -2.88 & -2.87 & -2.42 & $-6.49 * *$ & $-36.20^{* *}$ & $-24.47 * *$ \\
\hline $7.14^{* *}$ & $-18.90^{* * *}$ & -1.49 & -5.03 & 2.30 & $-7.40 * *$ & $-33.15^{* *}$ & $-30.45 * *$ \\
\hline $3.57^{*}$ & $-21.57 * *$ & -1.52 & $-6.48^{*}$ & 1.01 & $-7.40 * *$ & $-42.36 * *$ & $-47.47 * *$ \\
\hline $12.50^{* *}$ & $21.65^{* *}$ & $5.45^{*}$ & 4.32 & $6.70 * *$ & -4.17 & $-69.23 * *$ & $-69.02 * *$ \\
\hline$-5.26 * *$ & $-51.34 * *$ & $-4.84^{*}$ & $-15.11^{* *}$ & -3.43 & $-15.28 * *$ & $-75.33 * *$ & $-89.63 * *$ \\
\hline $13.85^{* *}$ & 0.00 & $-13.04 * *$ & $-13.66^{* *}$ & $-4.86^{* *}$ & $-13.89 * *$ & $-59.08 * *$ & -51.20 ** \\
\hline $3.61 *$ & $16.22^{* * *}$ & -4.05 & 2.16 & $-6.52 * *$ & -3.71 & $-31.32 * *$ & $-3.86 * *$ \\
\hline $39.76^{* *}$ & $56.77^{* *}$ & -0.68 & 4.32 & -0.44 & 3.71 & $-11.15^{* *}$ & $13.03 * *$ \\
\hline$-15.89 * *$ & $21.65^{\text {** }}$ & $-4.95^{*}$ & 3.60 & -0.90 & 1.39 & $6.88^{* *}$ & $46.28 * *$ \\
\hline $16.92 * *$ & 2.76 & -1.45 & -2.16 & $-7.16^{* *}$ & $-6.94 * *$ & $-58.11 * *$ & $-67.42^{* * *}$ \\
\hline $28.26^{* *}$ & $59.53^{* *}$ & -3.95 & 5.05 & -0.67 & 2.32 & $-26.72 * *$ & $13.83^{* *}$ \\
\hline-2.70 & -2.68 & -0.71 & 0.73 & -0.70 & -1.85 & $12.01 * *$ & $26.33 * *$ \\
\hline$-8.16^{* *}$ & $21.65^{* *}$ & -3.75 & 1.45 & 0.24 & -2.78 & $-5.76^{* *}$ & $15.43^{* * *}$ \\
\hline $3.57^{*}$ & $-21.57 * *$ & -2.26 & $-6.48^{*}$ & $-3.90 *$ & $-8.79 * *$ & $-3.81 * *$ & $-38.96 * *$ \\
\hline $30.12 * *$ & $45.99 * *$ & $-8.84 * *$ & -3.58 & -1.42 & -3.71 & $-9.25^{* *}$ & $27.93 * *$ \\
\hline $6.12 * *$ & $40.55^{* *}$ & 1.73 & 5.76 & $5.66^{* *}$ & 3.71 & $1.72 * *$ & $11.44^{* *}$ \\
\hline $7.14 * *$ & $-18.90 * *$ & 0.00 & -5.74 & 2.65 & -1.39 & $17.78^{* *}$ & $-40.43^{* *}$ \\
\hline $3.61 *$ & $16.22^{* *}$ & 1.38 & $5.76^{*}$ & 3.51 & 2.32 & 0.02 & $28.19 * *$ \\
\hline $15.00^{* *}$ & $24.33^{* * *}$ & -1.83 & -3.58 & -2.70 & $-8.33^{* *}$ & $-1.58 * *$ & $-40.69 * *$ \\
\hline $10.28 * *$ & $59.53^{\text {** }}$ & $-11.63^{* *}$ & -4.32 & $-4.06^{*}$ & $-6.94 * *$ & $9.94 * *$ & $51.46^{* * *}$ \\
\hline $23.08^{* *}$ & $8.11^{\text {*** }}$ & -1.46 & -2.87 & 1.46 & -3.71 & $-23.94 * *$ & $-40.03^{* *}$ \\
\hline
\end{tabular}

$* \& * *$ Significance at $1 \%$ and $5 \%$, respectively; $\mathrm{MPH}=$ Mid parent heterosis; $\mathrm{SPH}=$ Standard parent heterosis, NFF

- Node no. of first female flower, DFF-Days to first female flower anthesis and DFH-Days to first fruit harvest

Table.2 Heterosis (\%) over mid parent and standard check for yield related traits in bitter gourd

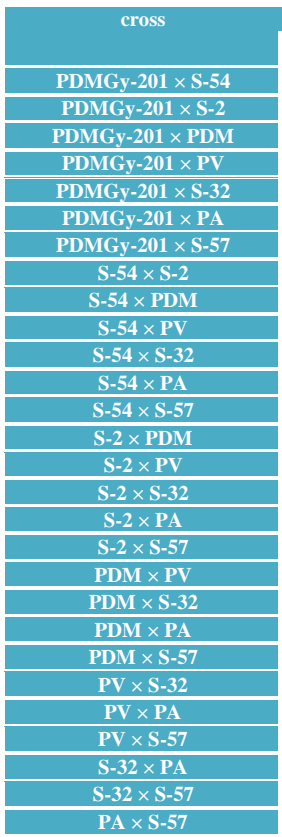

\begin{tabular}{|c|c|c|c|c|c|}
\hline \multicolumn{2}{|c|}{ FL $(\mathrm{cm})$} & \multicolumn{2}{|c|}{ FD (cm) } & \multicolumn{2}{|c|}{ FTH (mm) } \\
\hline MPH & SPH & MPH & SPH & MPH & SPH \\
\hline $14.91 * *$ & $10.53^{* * *}$ & $6.52 * *$ & $10.61^{* *}$ & $20.40^{* * *}$ & $16.63 * *$ \\
\hline$-23.92 * *$ & $-11.45^{* *}$ & $-4.55^{* *}$ & $-20.99 * *$ & $-2.82 * *$ & $-13.14 * *$ \\
\hline $3.53 * *$ & $6.10 * *$ & $11.69 * *$ & $-2.93 * *$ & $36.47 * *$ & $12.44 * *$ \\
\hline $20.73 * *$ & $10.28 * *$ & $-1.52^{* * *}$ & $-2.26 * *$ & $7.78^{* *}$ & $4.65^{* * *}$ \\
\hline$-10.70 * *$ & $-7.02 * *$ & $10.12 * *$ & $2.26^{* *}$ & $18.85^{* *}$ & $12.44 * *$ \\
\hline-1.59 & $-5.01 * *$ & $6.96^{* * *}$ & $-7.45 * *$ & $15.56^{* *}$ & 0.81 \\
\hline-2.13 & $-17.04 * *$ & $15.89 * *$ & $-6.77 * *$ & $40.38^{* *}$ & $13.14 * *$ \\
\hline 2.25 & $32.83^{* *}$ & $-6.84 * *$ & $-18.06 * *$ & $1.05^{* * *}$ & $-6.98^{* * *}$ \\
\hline-1.80 & $13.87^{* * *}$ & $9.39^{* *}$ & 0.90 & $14.81^{* *}$ & $-2.33^{* *}$ \\
\hline $13.68 * *$ & $19.21 * *$ & $-6.47 * *$ & $-2.26^{* * *}$ & $-2.52 * *$ & $-2.67 * *$ \\
\hline$-18.58 * *$ & $-4.18 *$ & $9.58^{* *}$ & $7.67 * *$ & 0.80 & $-1.98 * *$ \\
\hline$-11.90 * *$ & -3.09 & $13.11 * *$ & $3.84 * *$ & $7.76^{* * *}$ & $-3.14 * *$ \\
\hline $33.43 * *$ & $31.16^{* * *}$ & $34.21 * *$ & $15.12^{* *}$ & $54.88^{* *}$ & $29.07 * *$ \\
\hline $6.75^{* *}$ & $45.36 * *$ & $15.34 * *$ & $-18.06 * *$ & $13.50^{* *}$ & $-11.98 * *$ \\
\hline $6.90 * *$ & $33.67 * *$ & $13.51 * *$ & $-5.19 * *$ & $9.24 * *$ & 0.81 \\
\hline$-17.98 * *$ & 13.03 ** & $-9.27 * *$ & $-30.02 * *$ & $-1.51 * *$ & $-11.63 * *$ \\
\hline-0.11 & $30.08 * *$ & $10.64 * *$ & $-21.67 * *$ & -0.24 & $-17.79 * *$ \\
\hline $8.11^{* *}$ & $28.07 * *$ & $13.95^{* * *}$ & $-26.19 * *$ & $7.93 * *$ & $-18.26 * *$ \\
\hline $12.53 * *$ & $25.06^{* *}$ & $1.29 * *$ & $-11.29 *$ & $14.55^{* *}$ & $-2.33^{* *}$ \\
\hline $8.54 * *$ & $34.50^{* * *}$ & $16.67^{* * *}$ & $-5.19 * *$ & $10.54 * *$ & $-8.49 * *$ \\
\hline$-3.23^{*}$ & $12.53^{* *}$ & $-2.51 * *$ & $-27.09 * *$ & $9.51^{* *}$ & $-17.44 * *$ \\
\hline $21.70^{* * *}$ & $27.23^{* * *}$ & $32.24 * *$ & $-9.03 * *$ & $40.85^{* *}$ & $-3.14 * *$ \\
\hline 0.00 & $12.78^{* * *}$ & $-2.81 * *$ & $-9.03 * *$ & -0.60 & $-3.14 * *$ \\
\hline-1.19 & $3.84^{*}$ & 0.00 & $-12.64 * *$ & $-3.66 * *$ & $-13.14 * *$ \\
\hline 0.75 & $-5.85^{* *}$ & $-2.78 * *$ & $-20.99 * *$ & $-1.62 * *$ & $-17.79 * *$ \\
\hline $25.15^{* *}$ & $47.62^{* * *}$ & $14.42 * *$ & $-7.45^{* *}$ & $10.62^{* * *}$ & $-3.14 * *$ \\
\hline $7.99 * *$ & $14.70^{* * *}$ & $40.70^{* * *}$ & $5.42 * *$ & $10.05^{* *}$ & $-10.81 * *$ \\
\hline $11.30 * *$ & $9.69 * *$ & $31.87 * *$ & $-9.71 * *$ & $42.11^{* *}$ & $4.65 * *$ \\
\hline
\end{tabular}

\begin{tabular}{|r|r|}
\hline \multicolumn{2}{|c|}{ NFP } \\
\hline MPH & \multicolumn{1}{|c|}{ SPH } \\
\hline$-6.78^{* *}$ & -0.89 \\
\hline $9.86^{* *}$ & $5.41^{*}$ \\
\hline $11.34^{* *}$ & -2.70 \\
\hline $16.81^{* *}$ & $18.92^{* *}$ \\
\hline 1.94 & $-5.41^{*}$ \\
\hline $11.02^{* *}$ & $18.03^{* *}$ \\
\hline-2.59 & 1.81 \\
\hline $7.76^{* *}$ & $6.30^{* *}$ \\
\hline $11.00^{* *}$ & 0.00 \\
\hline-1.72 & 2.70 \\
\hline $5.66^{* *}$ & 0.89 \\
\hline $4.96^{* *}$ & $14.41^{* *}$ \\
\hline$-10.92^{* *}$ & $-4.51^{*}$ \\
\hline $22.03^{* *}$ & -2.70 \\
\hline $7.18^{* *}$ & 0.89 \\
\hline $17.46^{* *}$ & 0.00 \\
\hline $19.63^{* *}$ & $18.03^{* *}$ \\
\hline $6.05^{*}$ & 2.70 \\
\hline $16.84^{* *}$ & 0.00 \\
\hline $7.06^{* *}$ & $-18.03^{* *}$ \\
\hline $14.00^{* *}$ & 2.70 \\
\hline $11.22^{* *}$ & -1.81 \\
\hline $5.94^{* *}$ & -3.59 \\
\hline $5.17^{* *}$ & $9.92^{* *}$ \\
\hline $4.39^{* *}$ & 7.22 \\
\hline-0.94 & $-5.41^{*}$ \\
\hline $5.77^{* *}$ & -0.89 \\
\hline 1.68 & $9.00^{* *}$ \\
\hline 0.6 & $-{ }^{*}$ \\
\hline
\end{tabular}

\begin{tabular}{|c|}
\hline MPH \\
\hline $75.83^{* *}$ \\
\hline $14.89^{* *}$ \\
\hline $28.39 * *$ \\
\hline $47.94 * *$ \\
\hline $36.42 * *$ \\
\hline $18.67 * *$ \\
\hline $36.40 * *$ \\
\hline $13.47 * *$ \\
\hline $29.82 * *$ \\
\hline $30.03^{* *}$ \\
\hline $34.81 * *$ \\
\hline $35.08^{* *}$ \\
\hline $67.72 * *$ \\
\hline $11.76 * *$ \\
\hline $9.10^{* * *}$ \\
\hline$-6.68 * *$ \\
\hline $13.33^{* *}$ \\
\hline 6.63 \\
\hline $12.13^{* *}$ \\
\hline $13.64 * *$ \\
\hline-4.35 \\
\hline-4.20 \\
\hline $15.88^{* *}$ \\
\hline $10.50^{* * *}$ \\
\hline $15.09 * *$ \\
\hline $16.27 * *$ \\
\hline $\begin{array}{l}18.63^{* *} \\
24.73^{* *}\end{array}$ \\
\hline
\end{tabular}

\begin{tabular}{|l|l|l|}
\hline & \multicolumn{2}{|c|}{ YLD/P (kg) } \\
\hline SPH & MPH & SPH \\
\hline $46.77^{* *}$ & $56.75^{* *}$ & $46.67^{* *}$ \\
\hline $26.73^{* *}$ & $24.49^{* *}$ & $34.44^{* *}$ \\
\hline $56.30^{* *}$ & $46.87^{* *}$ & $53.89^{* *}$ \\
\hline $39.26^{* *}$ & $67.50^{* *}$ & $67.22^{* *}$ \\
\hline $38.38^{* *}$ & $35.88^{* *}$ & $31.67^{* *}$ \\
\hline $22.84^{* *}$ & $28.29^{* *}$ & $46.11^{* *}$ \\
\hline $31.29^{* *}$ & $27.80^{* *}$ & $33.89^{* *}$ \\
\hline $19.69^{* *}$ & $25.59^{* *}$ & $28.33^{* *}$ \\
\hline $51.80^{* *}$ & $54.64^{* *}$ & $52.78^{* *}$ \\
\hline $16.16^{* *}$ & $27.79^{* *}$ & $19.44^{* *}$ \\
\hline $30.27^{* *}$ & $45.62^{* *}$ & $32.22^{* *}$ \\
\hline $33.33^{* *}$ & $41.92^{* *}$ & $52.78^{* *}$ \\
\hline $53.37^{* *}$ & $49.02^{* *}$ & $47.22^{* *}$ \\
\hline $60.67^{* *}$ & $37.90^{* *}$ & $56.67^{* *}$ \\
\hline $26.73^{* *}$ & $20.24^{* *}$ & $30.56^{* *}$ \\
\hline $15.19^{* *}$ & $9.81^{* *}$ & $16.11^{* *}$ \\
\hline $42.27^{* *}$ & $37.46^{* *}$ & $68.33^{* *}$ \\
\hline $26.11^{* *}$ & $14.43^{* *}$ & $30.00^{* *}$ \\
\hline $43.09^{* *}$ & $36.80^{* *}$ & $43.89^{* *}$ \\
\hline $53.31^{* *}$ & $23.40^{* *}$ & $26.67^{* *}$ \\
\hline $31.02^{* *}$ & $13.58^{* *}$ & $35.56^{* *}$ \\
\hline $24.27^{* *}$ & $10.91^{* *}$ & $22.22^{* *}$ \\
\hline $24.34^{* *}$ & $24.07^{* *}$ & $20.56^{* *}$ \\
\hline $20.86^{* *}$ & $16.46^{* *}$ & $32.78^{* *}$ \\
\hline $17.53^{* *}$ & $20.42^{* *}$ & $26.67^{* *}$ \\
\hline $35.64^{* *}$ & $15.78^{* *}$ & $28.89^{* *}$ \\
\hline $29.80^{* *}$ & $26.29^{* *}$ & $29.44^{* *}$ \\
\hline $39.06^{* *}$ & $27.70^{* *}$ & $52.22^{* *}$ \\
\hline
\end{tabular}

$* \& * *$ Significance at $1 \%$ and $5 \%$, respectively; MPH $=$ Mid parent heterosis; SPH $=$ Standard parent heterosis, FL-

Fruit length, FD-Fruit diameter, FTH- Flesh thickness, NFP-Number of fruits per plant, AFW-Average fruit weight, YLD/P-Yield per plant. 
Table.3 Range of mean values for different traits of parents, $F_{1}$ hybrids and heterosis (over mid and standard parent)

\begin{tabular}{|c|c|c|c|c|c|c|c|c|c|c|}
\hline Particulars & NFF & DFF & DFH & Sex ratio $\mathrm{m} / \mathrm{f}$ & FL $(\mathbf{c m})$ & FD $(\mathrm{cm})$ & FTH $(\mathbf{m m})$ & NFP & AFW (g) & YLD/P (kg) \\
\hline \multicolumn{11}{|l|}{ Range of mean values } \\
\hline Parent & 4.67 to 20.33 & $\begin{array}{l}37 \text { to } \\
51\end{array}$ & $\begin{array}{l}57.33 \text { to } \\
78\end{array}$ & $\begin{array}{c}0(100 \% \text { female }) \\
\text { to } \\
11.75\end{array}$ & $\begin{array}{c}9.90 \text { to } \\
17.97\end{array}$ & 2.77 to 4.83 & 5.77 to 8.60 & 26.33 to 40.33 & 38.47 to 75.90 & $\begin{array}{l}1.57 \\
\text { to } \\
2.31\end{array}$ \\
\hline F1 & 6 to 19.67 & $\begin{array}{c}29.67 \text { to } \\
49\end{array}$ & 51.67 to 74.67 & 0.07 to 11.39 & $\begin{array}{c}9.93 \text { to } \\
17.67\end{array}$ & 3.10 to 5.10 & 7.03 to 11.10 & 30.33 to 44 & 56.33 to 78.57 & 2.09 to 3.03 \\
\hline \multicolumn{11}{|l|}{ Range of heterosis \% over } \\
\hline MP & $\begin{array}{c}-15.89 \text { to } \\
106.06\end{array}$ & -32.58 to 5.45 & -23.65 to 6.70 & -95.93 to 34.78 & $\begin{array}{l}-23.92 \text { to } \\
33.43\end{array}$ & -6.84 to 40.70 & -3.66 to 44.88 & $\begin{array}{l}-10.92 \text { to } \\
22.03\end{array}$ & -6.68 to 75.83 & 9.81 to 67.50 \\
\hline SP & $\begin{array}{c}-51.34 \text { to } \\
59.53\end{array}$ & -35.96 to 5.76 & -28.24 to 3.71 & -99.07 to 51.46 & $\begin{array}{c}-17.04 \text { to } \\
47.62\end{array}$ & $\begin{array}{c}-30.02 \text { to } \\
15.12\end{array}$ & $\begin{array}{c}-18.26 \text { to } \\
29.07\end{array}$ & $\begin{array}{c}-18.03 \text { to } \\
18.92\end{array}$ & 15.19 to 60.67 & 16.11 to 68.33 \\
\hline \multicolumn{11}{|l|}{$\begin{array}{l}\text { No. of significant desirable } \\
\text { heterotic crosses over }\end{array}$} \\
\hline MP & 03 & 24 & 20 & 21 & 14 & 19 & 20 & 21 & 24 & 28 \\
\hline SP & 10 & 19 & 23 & 19 & 20 & 06 & 07 & 07 & 28 & 28 \\
\hline \multirow[t]{3}{*}{$\begin{array}{l}\text { Three top parents with their } \\
\text { mean values }\end{array}$} & $\begin{array}{l}\text { PDMGy-201 } \\
(4.67)\end{array}$ & $\begin{array}{l}\text { PDMGy-201 } \\
\text { (37) }\end{array}$ & $\begin{array}{l}\text { PDMGy-201 } \\
\text { (57) }\end{array}$ & $\begin{array}{l}\text { PDMGy-201 (0) } \\
\text { (100\% female) }\end{array}$ & $\begin{array}{c}\text { S-2 } \\
(17.97)\end{array}$ & $\mathrm{S}-54(4.83)$ & PV (8.60) & $\begin{array}{c}\text { S-54, PA } \\
(40.33)\end{array}$ & PDM (75.90) & PA (2.31) \\
\hline & $\begin{array}{l}\text { S-54, S-32 } \\
\quad(6.33)\end{array}$ & S-54 (41.67) & S-54 (60) & PA $(0.10)$ & $\begin{array}{c}\text { S-32 } \\
(15.03)\end{array}$ & PV (4.43) & S-54 (8.57) & S-57 (39) & S-2 (60.70) & S-2 (2.10) \\
\hline & $\begin{array}{l}\text { PDM, PV } \\
(12.33)\end{array}$ & PA (41) & PA (66.33) & S-54 (6.20) & $\begin{array}{l}\text { PDM } \\
(14.63)\end{array}$ & $\begin{array}{c}\text { PDMGy-201 } \\
(4.37)\end{array}$ & $\mathrm{S}-32(8.17)$ & $\begin{array}{l}\text { PDMGy-201 } \\
(38.33)\end{array}$ & PA (58.07) & $\begin{array}{l}\text { PDM, S-57 } \\
(1.99)\end{array}$ \\
\hline \multirow{4}{*}{$\begin{array}{l}\text { Three top F1 hybrids with } \\
\text { heterosis\% over MP }\end{array}$} & & & & & & & & & & \\
\hline & $\begin{array}{c}\text { S- } 2 \times \text { S-32(- } \\
15.89)\end{array}$ & $\begin{array}{l}\text { PDMGy-201 } \\
\times \text { S-2 } \\
\quad(-32.58)\end{array}$ & $\begin{array}{l}\text { PDMGy-201 } \\
\times \text { S-2 } \\
\quad(-23.65)\end{array}$ & $\begin{array}{c}\text { PDMGy-201 } \times \text { S-2 } \\
(-95.93)\end{array}$ & $\begin{array}{l}\text { S-54 × S- } \\
57 \\
\quad(33.43)\end{array}$ & $\begin{array}{c}\text { S-32 } \times \text { S-57 } \\
\quad(40.7)\end{array}$ & $\begin{array}{l}\text { S-54 × S-57 } \\
\quad(54.88)\end{array}$ & $\begin{array}{l}\text { S-2 } 2 \text { PDM } \\
\quad(22.03)\end{array}$ & $\begin{array}{l}\text { PDMGy-201 } \\
\times \text { S-54 } \\
\quad(75.83)\end{array}$ & $\begin{array}{l}\text { PDMGy-201 } \\
\times \text { PV } \\
\quad(67.50)\end{array}$ \\
\hline & $\begin{array}{l}\mathrm{PDM} \times \mathrm{S}-32 \\
\quad(-8.16)\end{array}$ & $\begin{array}{l}\text { PDMGy-201 } \\
\times \text { PDM } \\
\quad(-25.60)\end{array}$ & $\begin{array}{l}\text { PDMGy-201 } \\
\times \text { PV } \\
\quad(-17.53)\end{array}$ & $\begin{array}{l}\mathrm{S}-54 \times \mathrm{PA} \\
\quad(-75.33)\end{array}$ & $\begin{array}{l}\text { S-32 } 3 \text { PA } \\
\quad(25.17)\end{array}$ & $\begin{array}{l}\text { S-54 } \times \text { S-57 } \\
\quad(34.21)\end{array}$ & $\begin{array}{r}\mathrm{PA} \times \mathrm{S}-57 \\
(42.11)\end{array}$ & $\begin{array}{l}\text { S-2 } \\
\quad(19.63)\end{array}$ & $\begin{array}{l}\text { S- } 54 \times \text { S- } 57 \\
\quad(67.72)\end{array}$ & $\begin{array}{l}\text { PDMGy-201 } \\
\times \text { S-54 } \\
\quad(56.75)\end{array}$ \\
\hline & $\begin{array}{l}\text { S-54 } \times \text { PA } \\
\quad(-5.26)\end{array}$ & $\begin{array}{l}\text { PDMGy-201 } \\
\times \text { S-32 } \\
(-14.18)\end{array}$ & $\begin{array}{l}\text { PDMGy-201 } \\
\times \text { PDM } \\
\quad(-9.66)\end{array}$ & $\begin{array}{l}\text { PDMGy- } 201 \times \text { S- } \\
57 \quad(-71.24)\end{array}$ & $\begin{array}{l}\text { PDM } \times \text { S }- \\
57 \\
\quad(21.7)\end{array}$ & $\begin{array}{l}\mathrm{PDM} \times \mathrm{S}-57 \\
(32.24)\end{array}$ & $\begin{array}{l}\mathrm{PDM} \times \mathrm{S}-57 \\
\quad(40.85)\end{array}$ & $\begin{array}{r}\text { S-2 } \\
(17.46)\end{array}$ & $\begin{array}{l}\text { PDMGy-201 } \\
\times \text { PV } \\
\quad(47.94)\end{array}$ & $\begin{array}{c}\text { S- } 54 \times \text { PDM } \\
(54.64)\end{array}$ \\
\hline \multirow{4}{*}{$\begin{array}{l}\text { Three top F1 hybrids with } \\
\text { heterosis \% over SP }\end{array}$} & & & & & & & & & & \\
\hline & $\begin{array}{l}\text { S- } 54 \times \text { PA }(- \\
51.34)\end{array}$ & $\begin{array}{l}\text { PDMGy-201 } \\
\times \text { S-2 } \\
\quad(-35.96)\end{array}$ & $\begin{array}{l}\text { PDMGy-201 } \\
\times \text { S-2 } \\
\quad(-28.24)\end{array}$ & $\begin{array}{c}\text { PDMGy-201 } \times \text { PA } \\
(-99.07)\end{array}$ & $\begin{array}{l}\text { S- } 32 \times \text { S- } \\
57 \\
(47.62)\end{array}$ & $\begin{array}{l}\text { S- } 54 \times \text { S- } 57 \\
\quad(15.12)\end{array}$ & $\begin{array}{l}\text { S-54 } \times \text { S-57 } \\
\quad(29.07)\end{array}$ & $\begin{array}{l}\text { PDMGy-201 } \\
\times \text { PV } \\
\quad(18.92)\end{array}$ & $\begin{array}{r}\text { S-2 } 2 \times \text { PDM } \\
\quad(60.67)\end{array}$ & $\begin{array}{l}\mathrm{S}-2 \times \mathrm{PA} \\
\quad(68.33)\end{array}$ \\
\hline & $\begin{array}{c}\text { PDMGy-201 } \\
\times \text { S-54 } \\
(-27.01)\end{array}$ & $\begin{array}{l}\text { PDMGy-201 } \\
\times \text { PV } \\
\quad(-33.09)\end{array}$ & $\begin{array}{l}\text { PDMGy-201 } \\
\times \text { PV } \\
\quad(-25.93)\end{array}$ & $\begin{array}{c}\text { PDMGy-201 × S-2 } \\
(-96.81)\end{array}$ & $\begin{array}{l}\text { S-2 } \times \\
\text { PDM } \\
(45.36)\end{array}$ & $\begin{array}{l}\text { PDMGy-201 } \\
\times \text { S-54 } \\
\quad(10.61)\end{array}$ & $\begin{array}{l}\text { PDMGy-201 } \\
\times \text { S-54 } \\
\quad(16.63)\end{array}$ & $\begin{array}{l}\text { PDMGy-201 } \\
\times \text { PA } \\
\quad(18.03)\end{array}$ & $\begin{array}{l}\text { PDMGy-201 } \\
\times \text { PDM } \\
\quad(56.30)\end{array}$ & $\begin{array}{l}\text { PDMGy-201 } \\
\times \text { PV } \\
\quad(67.22)\end{array}$ \\
\hline & $\begin{array}{l}\text { S-54 } 5 \text { PV } \\
(-21.57)\end{array}$ & $\begin{array}{l}\text { PDMGy-201 } \\
\times \text { S-54 } \\
\quad(-25.90)\end{array}$ & $\begin{array}{l}\text { PDMGy-201 } \\
\times \text { S-54 } \\
(-22.22)\end{array}$ & $\begin{array}{l}\text { S-54 } \times \text { PA } \\
\quad(-89.63)\end{array}$ & $\begin{array}{l}\text { PDM } \times \text { S }- \\
32 \\
(34.50)\end{array}$ & $\begin{array}{l}\text { S- } 54 \times \text { S-32 } \\
(7.67)\end{array}$ & $\begin{array}{l}\text { PDMGy-201 } \\
\times \text { S-57 } \\
\quad(13.14)\end{array}$ & $\begin{array}{l}\mathrm{S}-2 \times \mathrm{P}_{7} \\
\quad(18.03)\end{array}$ & $\begin{array}{l}\text { S- } 54 \times \text { S- } 57 \\
\quad(53.37)\end{array}$ & $\begin{array}{c}\mathrm{S}-2 \times \mathrm{PDM} \\
\quad(56.67)\end{array}$ \\
\hline
\end{tabular}

NFF -Node no. of first female flower, DFF-Days to first female flower anthesis, DFH-Days to first fruit harvest, FL-Fruit length, FD-Fruit diameter, FTH- Flesh thickness, NFP-Number of fruits per plant, AFW-Average fruit weight, YLD/P-Yield per plant, MP = Mid parent; SP = Standard parent $(\mathrm{Pusavisesh})$ 
Three top combinations has either of the gynoecious parent PDMGy-201 or predominantly gynoecious Pusa Aushadhi showed maximum heterosis in negative direction for sex ratio, PDMGy-201 $\times$ S-2 (95.93\%), S-54 × PA (-75.33\%) and PDMGy$201 \times$ S-57 $(-71.24 \%)$ over mid parent and PDMGy-201 × PA (-99.07\%), PDMGy-201 × S-2 (-96.81\%) and S-54 $\times$ PA $(-89.63 \%)$ over standard parent. Similar results were also reported earlier in bitter gourd by Behera et al., (2009) and Dey et al., (2010).

Out of twenty eight hybrids 14 over mid parent and 20 over standard parent showed significant desirable heterosis for fruit length. The highest standard heterosis for fruit length was observed in the hybrid S-32 $\times$ S-57 (47.62\%) followed by $\mathrm{S}-2 \times \mathrm{PDM}(45.36 \%)$ and $\mathrm{PDM} \times \mathrm{S}-32$ $(34.50 \%)$. Whereas, maximum positive heterosis over mid parent was observed in the cross S-54 $\times$ S-57 (33.43\%) followed by S-32 $\times$ PA $(25.17 \%)$ and PDM $\times$ S-57 (21.7\%). However, for fruit diameter 19 and 6 crosses out of $28 F_{1}$ hybrids showed significant and positive heterosis over their respective mid and standard parent. Maximum positive heterosis over the mid parent was observed in the cross S-32 × S-57 (40.7\%) followed by S-54 × S-57 $(34.21 \%)$ and PDM $\times$ S-57 (32.24\%). The highest standard heterosis was recorded in hybrid S-54 $\times$ S-57 (15.12\%) followed by PDMGy-201 × S-54 (10.61\%) and S-54 × S-57 (7.67\%).

Fruit flesh thickness is an important fruit quality trait and an essential determinant of yield in bitter gourd; the thicker the fruit flesh, the greater the edible portion of the fruit. Out of twenty eight crosses 20 and 7 hybrids showed significant and positive heterosis for fruit flesh thickness over mid and standard parent respectively. However, the highest significant and positive value for mid and standard parent heterosis was recorded in the cross S-54 $\times$ S-57 $(54.88 \%)$ and $(29.07 \%)$ respectively. For number of fruits per plant 21 and 7 crosses over their respective mid and standard parent exhibited significant and positive heterosis. The maximum heterosis for number of fruits per plant was observed in the cross S-2 $\times$ PDM $(22.03 \%)$ followed by S-2 $\times$ PA $(19.63 \%)$ and S-2 $\times$ S-32 (17.46\%) over mid parent and the cross PDMGy-201 $\times$ PV (18.92\%) followed by PDMGy-201 $\times$ PA and S-2 $\times$ PA $(18.03 \%)$ over standard parent. Twenty four crosses showed significant and positive heterosis over mid parent for average fruit weight. Whereas, standard parent heterosis was significant and positive for all crosses. Hybrids PDMGy-201 × S-54, S-54 × S-57 and PDMGy-201 $\times$ PV exhibited higher percentage of mid parent heterosis for average fruit weight (75.83\%), $(67.72 \%)$ and $(47.94 \%)$ respectively. Whereas, the three top crosses over standard parent were S-2 × PDM (60.67\%), PDMGy-201 × PDM $(56.30 \%)$ and S-54 $\times$ S-57 (53.37\%). These results were in conformity with those of Dey et al., (2012), Talekar et al., (2013) and AlMamuna et al., (2016).

All crosses showed significant and positive heterosis over mid and standard parent for total yield per plant. Hybrid PDMGy-201 × PV $(67.5 \%)$ recorded higher percentage of mid parent heterosis followed by PDMGy-201 × S$54(56.75 \%)$ and S-54 $\times$ PDM (54.64\%). However, maximum heterosis over standard parent was recorded in the cross S-2 $\times$ PA $(68.33 \%)$ followed by PDMGy-201 $\times$ PV (67.22\%) and S-2 $\times$ PDM (56.67\%). Our results revealed that characters such as fruit weight and number of fruits per plant were the major contributors towards increased yield per plant for these particular crosses as reported earlier by Dey et al., 2005 and Radha Rani et al., 2015. Based on the performance of $28 \mathrm{~F}_{1}$ hybrids three best performing hybrids were S- $2 \times \mathrm{PA}$, PDMGy-201 $\times$ PV and S- $2 \times$ PDM and these hybrids may be tested under multi-locational trials.

\section{References}

Alam, M.A., Uddin, R., Subhan, N., Rahman, M.M., Jain, P, and Reza, H.M. 2015. Beneficial Role of Bitter Melon Supplementation in Obesity and Related 
Complications in Metabolic Syndrome. Journal of Lipids 2015. doi.org/10.1155/2015/496169.

Al-Mamuna, M. H., Rashida, M. H., Uddina, M. N., Islama, M. R. and Asaduzzaman, Md. 2016. Heterosis studies in bitter gourd. International Journal of Vegetable Science doi.org/10.1080/19315260.2015.1072613.

Behera, T. K. 2004. Heterosis in bitter gourd. In: Singh, P.K., Dasgupta, S.K., Tripathi, S.K. (Eds.), Hybrid Vegetable Development. The Haworth Press. New York, USA, pp. 217 221.

Behera, T. K., Dey, S. S. and Sirohi, P. S. 2006. DBGy-201 and DBGy-202: two gynoecious lines in bitter gourd (Momordica charantia L.) isolated from indigenous source. Indian Journal of Genetics. 66: 61-62.

Behera, T. K., Dey, S. S., Munshi, A. D., Gaikwad, A. B., Pal, A. and Singh, I. 2009. Sex inheritance and development of gynoecious hybrids in bitter gourd (Momordica charantia L.). Scientia Horticulture 120: 130-133.

Budrat, P. and Shotipruk, A. 2008. Extraction of phenolic compounds from fruits of bitter melon (Momordica charantia) with subcritical water extraction and antioxidant activities of these extracts. Chiang Mai Journal of Science 35(1): 123-130.

Dey, S. S., Behera, T. K. and Munshi, A. D. 2010. Gynoecious inbred with better combining ability improves yield and earliness in bitter gourd (Momordica charantia L.). Euphytica. 173:37-47.

Dey, S. S., Behera, T. K., Munshi, A. D., Rakshit, S. and Bhatia, R. 2012. Utility of gynoecious sex form in heterosis breeding of bitter gourd and genetics of associated vegetative and flowering traits. Indian Journal of Horticulture 69(4): 523-529.
Dey, S. S., Behera, T. K., Pal, A. and Munshi, A. D. 2005. Correlation and path coefficient analysis in bitter gourd (Momordica charantia L.). Vegetable Science. 32(2): 173-176.

Gangadhara Rao, P, Behera T. K., Munshi A. D. and Dev B. 2018. Estimation of genetic components of variation and heterosis studies in bitter gourd for horticultural traits. Indian Journal of Horticulture 74(2): 227-232.

Joseph, B. and Jini, D. 2013. Antidiabetic effects of Momordica charantia (bitter melon) and its medicinal potency. Asian Pacific Journal of Tropical Disease, 3(2): 93-102.

Laxuman, S. A., Patil, P. M., Salimath, P. R., Dharmatti, A. S., Byadgi and Nirmalayenagi. 2012. Heterosis and combining ability analysis for productivity traits in bitter gourd (Momordica charantia L.). Karnataka Journal of Agricultural Sciences 25(1): 9-13.

Radha Rani, K., Raju, S and Reddy, K. R. 2015. Variability, correlation and path analysis studies in bitter gourd (Momordica charantia L.). Agricultural Science Digest 35(2): 106-110.

Radha Rani, K., Reddy, K. R. and Raju, S. 2014. Inheritance of yield and its related traits in bitter gourd (Momordica charantia L.). Molecular Plant Breeding 5(1): 1-4.

Ram, D., Kumar, S., Singh, M., Rai, M. and Kalloo, G. 2006. Inheritance of gynoecism in bitter gourd (Momordica charantia L.). Journal of Heredity. 97: 294-95.

Talekar. N. S., Vaddoria, M. A. and Kalkaran, G. V. 2013. Heterosis studies for quantitative traits in bitter gourd (Momordica charantia L.) Progressive Research. 8: 650-653.

\section{How to cite this article:}

Ahmad Alhariri, T.K. Behera, A.D. Munshi, C. Bharadwaj and Jat, G.S. 2018. Exploiting Gynoecious Line for Earliness and Yield Traits in Bitter Gourd (Momoredica charantia L.). Int.J.Curr.Microbiol.App.Sci. 7(11): 922-928. doi: https://doi.org/10.20546/ijcmas.2018.711.108 